\title{
Dynamic vibration absorption design and parameter analysis of oil tank wall of transformer
}

\author{
Hong Zhang ${ }^{1}$, Meigen $\mathrm{Cao}^{2 *}$ \\ ${ }^{1}$ Shanghai University of Electric Power, Pudong New District, Shanghai, 20090, China \\ ${ }^{2}$ North China University of Technology, Shijingshan District, Beijing, 100144, China
}

\begin{abstract}
Taking a $10 \mathrm{kV}$ test transformer as the research object, based on the analysis of transformer vibration and noise vibration sources, the oil tank model of the test transformer is established firstly, and the vibration mode analysis of the oil tank is carried out, and the typical vibration modes of transformer oil tanks with different thicknesses are obtained Then, according to the characteristics of vibration and vibration source of distribution transformer tank wall, the design method and parameter optimization analysis of transformer tank wall vibration absorption are carried out. The analysis shows that the principle of dynamic vibration absorption is clear, and the vibration of the box wall can be effectively controlled with $76 \%$ vibration reduction efficiency under the condition of reasonable arrangement of vibration absorption devices and parameter selection. The vibration control effect of vibration absorber increases with the increase of mass ratio between vibration absorber and transformer tank wall. When the mass ratio is determined, the closer the stiffness of vibration absorber is to the optimal stiffness, the better the vibration reduction effect will be.
\end{abstract}

\section{Introduction}

With the advancement of urbanization, substations go deep into load centers, and fixed equipment such as transformers in substations have become one of the main sources of urban noise. Environmental noise pollution caused by transformers and other facilities can not be ignored. The radiated noise of power transformer mainly comes from vibration of oil tank. In order to achieve the goal of power transformer vibration reduction and noise reduction, it is more effective and economical to suppress the vibration of oil tank and achieve the purpose of transformer noise reduction. At present, there are two main measures to reduce vibration and noise of transformer by restraining oil tank: one is to add sound insulation layer on the wall of transformer oil tank. Wang Yong damped the oil tank and ancillary structure of transformer to achieve the isolation effect of transformer vibration and noise $\mathrm{e}^{[1]}$. Li Hongkui and others put forward the method of painting damping layer on the outer surface of transformer oil tank and sticking sound insulation board to reduce the noise of transformer ${ }^{[2]} . \mathrm{Xu}$ Xingshuai proposed that steel plates should be added to the surface of transformer oil tank, and the air damping film formed between the steel plates and the oil tank wall should be used to reduce the vibration of oil $\operatorname{tank}^{[3]}$. Another method is to change the structure of transformer oil tank. Gu Xiao 'an et al. proposed to fill iron chips and sand between transformer reinforcing ribs to increase the weight of oil tank and reduce its vibration amplitude ${ }^{[4]}$.
Passive vibration absorption technology of transformer box wall mainly depends on dynamic vibration absorber, which is composed of three basic elements: auxiliary mass, spring and damping, and belongs to passive vibration absorber for suppressing vibration $^{[5]}$. Since its emergence, dynamic vibration absorbers have been widely used in the field of vibration reduction of buildings and machinery. In 1975, J.C.Snowdon proposed to use plate vibration absorber to control the vibration of the panel, which provided a basis for the application of dynamic vibration absorber in plate structure $^{[6]}$. T.Aida proposed to use beam, plate and shell dynamic vibration absorbers to control the multi-modal vibration of beams, plates and shells ${ }^{[7-8]}$. Since the 20th century, in view of the practical needs of engineering applications, dynamic vibration absorbers have appeared in many forms, such as disc type and piezoelectric type $^{[9]}$.

The purpose of this paper is to put forward a dynamic vibration absorption design scheme for transformer oil tank according to the vibration characteristics and dynamic characteristics of transformer oil tank surface, to suppress the vibration of transformer oil tank wall plate in the form of point distribution, and to provide reference for vibration reduction and noise reduction measures of transformer oil tank.

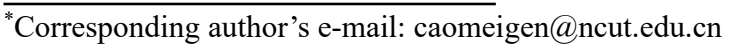




\section{Dynamic characteristics and modal analysis of tank wall of transformer.}

The root causes of transformer vibration are: core vibration caused by magnetostriction of silicon steel sheet; That is, when the core is excited, the size of silicon steel sheet increases along the direction of magnetic lines, while the size of silicon steel sheet decreases perpendicular to the direction of magnetic lines. This phenomenon is called magnetostriction. Magnetostriction makes the core vibrate periodically with the change of excitation frequency. There is electromagnetic attraction between the joints of silicon steel sheets and laminations due to magnetic leakage, which causes the vibration of the core; When the load current passes through the winding, the magnetic leakage caused by the load current causes the vibration of the winding and the oil tank wall $^{[10]}$.

In this paper, the three-dimensional modeling of transformer oil tank is carried out by using ANSYS software according to the size of $10 \mathrm{kV}$ test transformer oil tank. The transformer oil tank is made of Q235 steel, which is $1.3 \mathrm{~m}$ long, $0.6 \mathrm{~m}$ wide and $0.9 \mathrm{~m}$ high. In order to explore the dynamic characteristics of transformer oil tank wall and predict the weak parts of oil tank, modal analysis of transformer oil tank wall was carried out. In
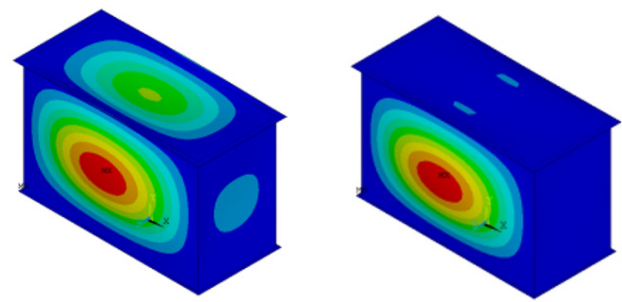

(a) The first mode $(27 \mathrm{~Hz})$

(b) Second mode $(30 \mathrm{~Hz})$

this paper, the structure of transformer oil tank has been simplified properly, the cooling fins and oil conservator on the surface of oil tank have been removed, the bottom of transformer oil tank has been set with fixed constraints, and the boundary condition of transformer oil tank wall plate has been set to be fixed on four sides. Figure 1 is a simplified transformer oil tank model.

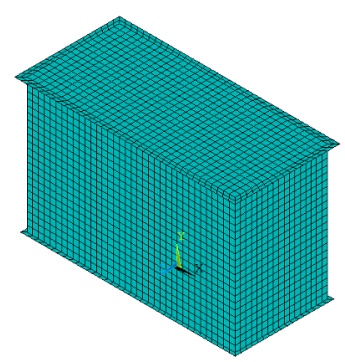

Fig 1.10 kVTransformer Tank Model

According to the theory of vibration correlation, higher-order modes have little influence on response and decay quickly. Low-order modes play a major role in vibration $^{[11]}$, so this paper mainly examines the first four modes for transformer modal analysis.

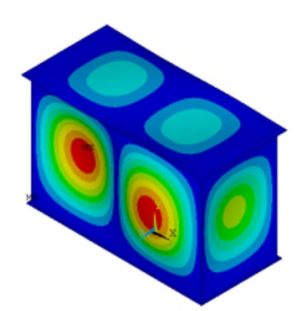

(c) The $3 \mathrm{rd}$ mode $(43 \mathrm{~Hz})$

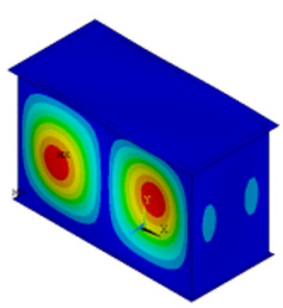

(d) The 4th mode $(46 \mathrm{~Hz})$

Fig 2. Modal analysis diagram of the first four modes of oil tank

The natural frequency of the first mode of the oil tank is about $27 \mathrm{~Hz}$, and its mode shape is that the central area of the long side wall of the oil tank bends obviously along the $\mathrm{z}$ axis; The natural frequency of the second mode is about $30 \mathrm{~Hz}$, and its mode shape is that the central area of the long side wall of the oil tank bends obviously along the $\mathrm{z}$ axis; The natural frequency of the third mode is about $43 \mathrm{~Hz}$, and its mode shape is that the areas on both sides of the center point of the long side wall of the oil tank bend along the $\mathrm{z}$ axis; The natural frequency of the fourth mode is about $46 \mathrm{~Hz}$, and its mode shape is that the areas on both sides of the long side wall of the oil tank bend obviously along the $\mathrm{z}$ axis;

From the modal analysis results, it can be seen that the short side wall of the oil tank bends less under the influence of vibration, while the long side wall of the oil tank bends more under the influence of vibration. The bending and torsion of the whole oil tank is obvious under the mode shape of the first-order mode, and the other order formations show the bending of the local part of the oil tank Most of the natural vibration modes on the oil tank wall are near the center point of the long side wall plate of the oil tank, which is also the best area for applying dynamic vibration absorbers.

\section{Vibration absorption design principle of oil tank wall}

The vibration reduction arrangement of dynamic vibration absorber on the surface of transformer oil tank is shown in Figure 3. The system composed of mass and spring is the main system, that is, the tank wall plate. Because the main material of the wall plate is Q235 steel, the damping factor of the wall plate is not considered. The system composed of mass M2, spring K2 and viscous damper $\mathrm{c}$ is a subsystem, namely damping vibration absorber.

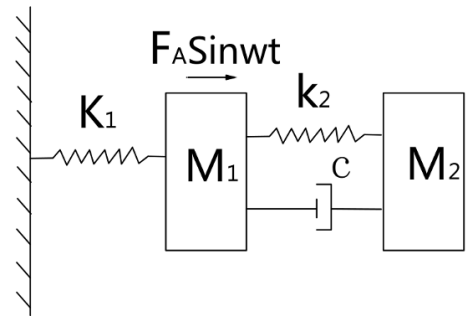

Fig. 3 Vibration model of main system with dynamic vibration absorber 
Based on the fixed-point theory ${ }^{[8]}$, it can be proposed to determine the specific parameters of the dynamic vibration absorbing device. The parameters of single dynamic vibration absorber are designed according to the principle of vibration absorption, and the mass of vibration absorber is determined according to the mass of main system:

$$
m_{2}=\mu m_{1}
$$

Determine the optimum spring stiffness of vibration absorber;

$$
k_{2}=\frac{k_{1} m_{2}}{m_{1}}\left(\frac{1}{1+\mu}\right)^{2}
$$

Determine the optimal damping coefficient of vibration absorber;

$$
\mathrm{c}=2 m_{2} \sqrt{\frac{k_{1}}{m_{1}}} \sqrt{\frac{3 \mu}{8(1+\mu)^{3}}}
$$

\section{Analysis of vibration absorption parameters of oil tank wall}

Matlab software simulation plug-in can build dynamic systems in many fields, including electronic circuits, shock absorbers and other electronic, mechanical and thermodynamic systems ${ }^{[12]}$. The simulation mainly includes two parts. Firstly, the model module diagram of the simulation system is established according to the mathematical relationship of the model, and then the parameter attributes of each module are set to make it simulate the dynamic system in a time period. According to the dynamic theory of two-degree-of-freedom vibration system, the dynamic vibration absorption simulation model of oil tank wall is established by simulink module.

When the vibration frequency of the oil tank is $100 \mathrm{~Hz}$, its central amplitude is shown in the following figure without installing a dynamic vibration absorber.

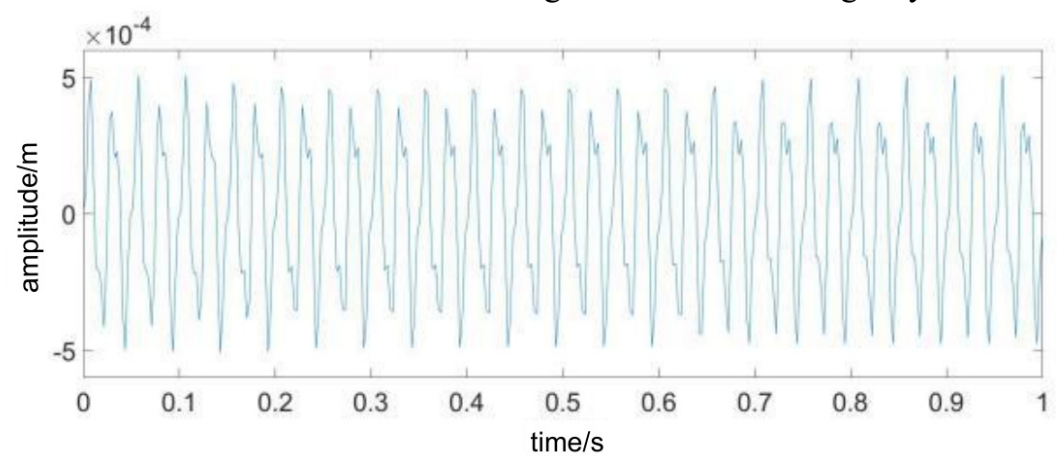

Fig. 4 Vibration history of the main system

According to the vibration absorption design principle formula in section 3, the optimal parameters of the dynamic vibration absorber with mass ratio, namely the corresponding optimal stiffness and damping, are determined. The vibration time history diagram of the tank wall and the vibration absorber applied is shown in the figure below.

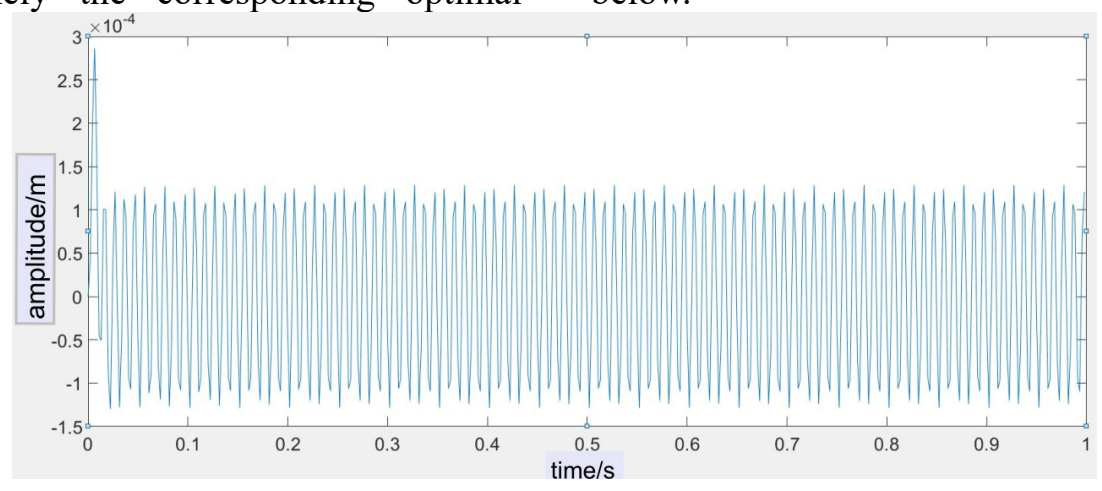

Fig 5. Vibration time history diagram of oil tank wall with vibration absorber

It can be seen from the above figure that after applying the vibration absorber, the vibration displacement of the oil tank wall plate is reduced from $5.12 \times 10^{-4} \mathrm{M}$ to about $1.25 \times 10^{-4} \mathrm{M}$, and the vibration absorption effect reaches $76 \%$, and the vibration of the transformer wall plate is significantly reduced, and the displacement of the vibration absorber is gradually stabilized in a small range for a period of time, and the vibration absorber has a good vibration absorption effect on the transformer oil tank wall.

By setting a series of mass ratios, the optimal stiffness and damping of its corresponding dynamic vibration absorber under the excitation of $100 \mathrm{~Hz}$ vibration source are calculated, and the amplitude of main system under the optimal design of corresponding mass ratio is simulated by simulation, and the influence law of mass ratio is observed. 


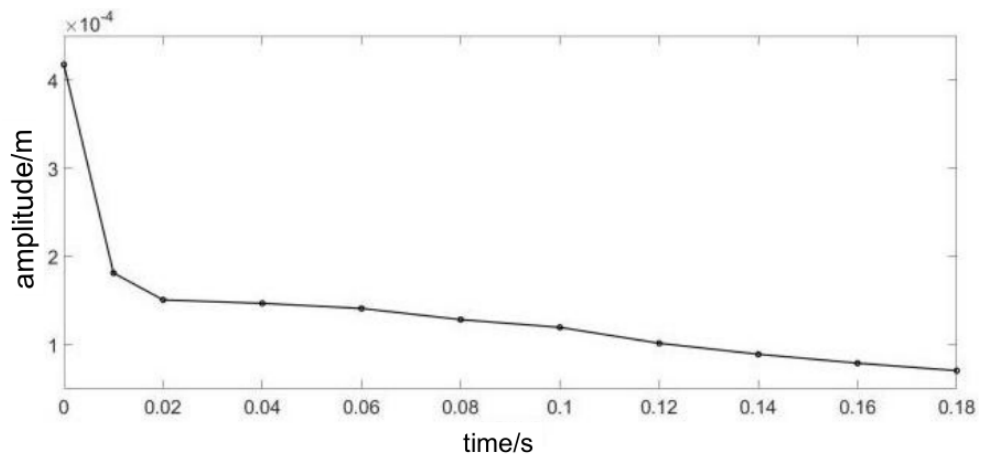

Fig 6. Variation of tank wall amplitude under different mass ratios

It can be seen from the figure that the amplitude of the main system gradually decreases with the increase of mass ratio. That is to say, increasing the mass ratio will increase the vibration control efficiency of the vibration absorber, but its mass will also increase. The mass ratio of dynamic vibration absorber should not exceed $20 \%$ for the structure of transformer oil tank wall plate.
In order to explore the influence of the stiffness of vibration absorber on the vibration reduction effect, a series of vibration absorber stiffnesses are set as $0 \mathrm{~N} / \mathrm{m}$, $100 \mathrm{~N} / \mathrm{m}, 200 \mathrm{~N} / \mathrm{m}, \quad 300 \mathrm{~N} / \mathrm{m}$ and $400 \mathrm{~N} / \mathrm{m}$, and the following figure is the amplitude response diagram of the oil tank panel under different stiffnesses.

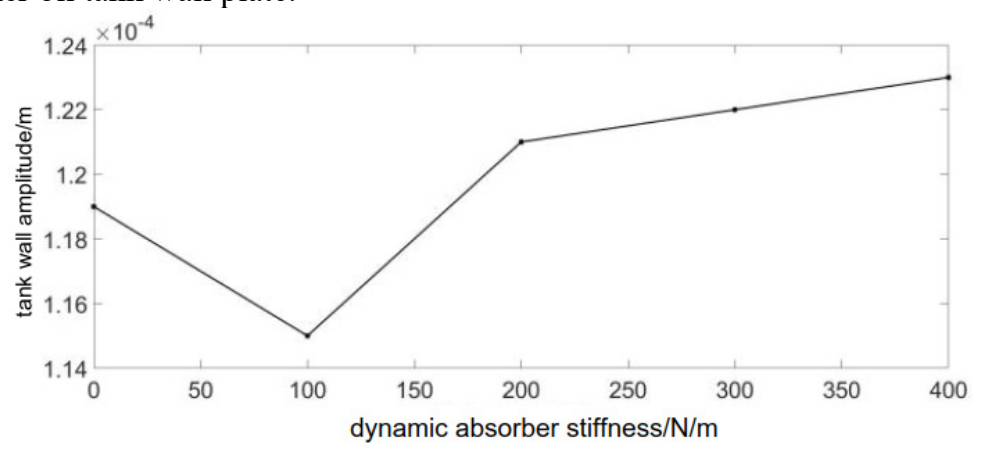

Fig .7. Response of fuel tank wall amplitude with different dynamic absorber stiffness

It can be seen from figure 7 that the amplitude of the tank wall is the smallest when the stiffness of the vibration absorber is $100 \mathrm{~N} / \mathrm{m}$, while it can be seen from table 2 that the stiffness of $100 \mathrm{~N} / \mathrm{m}$ is closest to the optimal stiffness, so when the mass ratio is determined, the closer the stiffness of the vibration absorber is to the optimal stiffness, the better the vibration reduction effect will be.

\section{5 conclusion}

In this paper, the vibration characteristics of $10 \mathrm{KV}$ transformer are obtained by vibration analysis. Combined with its vibration characteristics, the vibration modes of transformer with multiple frequencies are analyzed and vibration absorption design is carried out. Through parameter analysis of vibration absorption scheme by maltlab, the following conclusions are obtained:

(1) The vibration characteristics of transformer oil tank surface are mainly that the vibration energy of transformer oil tank is concentrated at several frequency points. The frequency point of transformer vibration energy concentration is $100 \mathrm{~Hz}$ which a series of integer multiples. For the vibration of transformer wall plate, multi-modal vibration control can be adopted. The vibration mode of vibration control object is determined by modal analysis, and several modes to be controlled are selected to establish corresponding vibration models, and independent parameter design can be carried out for each mode.

(2) According to the optimal design theory of the dynamic vibration absorber, the parameters of the dynamic vibration absorber are determined. Through simulation analysis, the vibration absorption efficiency reaches $76 \%$, and the variation trend of the amplitude of the oil tank wall under different mass ratios is obtained through parameter analysis. The amplitude of the oil tank wall decreases with the increase of the mass ratio, but the mass of the actual vibration absorption system should not be too large, and the best mass ratio range should be determined according to the actual situation. When the mass ratio is determined, the closer the stiffness of vibration absorber is to the optimal stiffness, the better the vibration reduction effect will be.

\section{References}

1. Wang Y, Ji ZY.2009, Analysis and control of the influence of transformer noise on indoor noise [J]. Environmental monitoring and early warning, 1(2):44-45.

2. Li Hk, Jing YT, Li Y, Li LN, Han FX.2016, Transformer design and electromagnetic analysis of ultra-high voltage double-body structure [J]. High 
Voltage Technology, 42(07): 2322-2328.

3. Xu XH.2016, analysis of noise sources and noise reduction countermeasures of oil-immersed power transformers $[\mathrm{J}]$. China new technology and new products, (03):79.

4. Gu XA Shen RY, Xu JT. 2001, Study on vibration and noise control methods of large power transformers $[\mathrm{J}]$. Noise and Vibration Control, (05):7-11.

5. Kazuto Seto, Ren MZ. 2013, Dynamic Vibration Absober and Its Application [M].

6. J.C.Snowdon,1995,Platet-Like Dynamic Vibration Absorber.[J]Journal of Engineering for Industry.97:88-93

7. T.Aida and T.Aso.1995,Vibration Contfol of Platc by a Plate-Type Dynamic Vibration Absorber[J]. Journal of Sound and Vibration,117(2): 332-338

8. T.Aida and T.Aso.1998,Vibration Contfol of Shallow Shell Structures Using a Shell-Type Dynamic Vibration Absorber[J], Journal of Sound and Vibration,218(2):245-267.

9. Wang ZJ., 2015, Design of Dynamic Vibration Absorber and Research on Low Frequency Sound Insulation Technology of Thin Plate Structure [D]. Beijing Jiaotong University.

10. YANG B. Stress, 2005, strain and structural dynamic[M]. Elsevier Academic.San Diego.

11. Lu SY, Li M, Ren RJ.2016, modal analysis of transformer based on finite element method[J]. mechanical engineer, (05):169-170.

12. Li Y, Zhu BL, Zhang W. 2004, Fundamentals of Modeling and Simulation of Dynamic Systems [M].Xidian University Press. 